\title{
Successive Parabolic Interpolation as Extremum Seeking Control for Microbial Fuel \& Electrolysis Cells
}

\author{
Tom R. Molderez ${ }^{* \ddagger}$, Bas de $\mathrm{Wit}^{\dagger}$, Korneel Rabaey ${ }^{\ddagger}$ and Marian Verhelst* \\ ${ }^{*}$ Department of Electrical Engineering (ESAT), KU Leuven, Leuven \\ ${ }^{\dagger}$ Department of Electrical Engineering, Eindhoven University of Technology, Eindhoven \\ ${ }_{\ddagger}^{\ddagger}$ Department of Biochemical and Microbial Technology, Ghent University, Ghent
}

\begin{abstract}
Microbial Fuel Cell (MFC) power production and Microbial Electrolysis Cell (MEC) organic production depend strongly on their dynamic environment conditions, like inlet substrate concentration, temperature, etc. This work presents a discrete extremum seeking controller to quickly tune the MFC and MEC electrical settings in order to achieve maximum performance irrespective of these dynamic environment conditions using the successive parabolic interpolation iteration scheme. The controller converges in about 3.5 days within $5 \%$ of the cell's maximum performance and in about 5.4 days within $1 \%$ for an established MFC model. The proposed discrete parabola controller converges $3 x$ faster than the state-of-theart controllers without requiring a time-consuming calibration procedure. Equally fast convergence speed is achieved on a MEC model.
\end{abstract}

\section{INTRODUCTION}

For a true circular economy, it is essential to recover the otherwise lost energy and chemicals in waste streams. Both are in an ever increasing rate consumed and only available in a finite amount. Microbial electrochemical technologies (METs) are a promising novel technology to process organic waste water streams. METs generate bio-electricity directly from organic waste in a microbial fuel cell (MFC) setup. Another possibility is a microbial electrolysis cell (MEC) configuration. This configuration uses the extracted energy to produce highvalue organics such as hydrogen gas with a minimal amount of external energy [1].

The current major drawback of MFCs is their low volumetric power density. Therefore, to make MFCs a competitive renewable technology, a lot of research focuses on the impact of the bioreactor structure, the used materials for the electrodes and the microbial community on the volumetric power density [2]. Recent MFCs achieve volumetric power densities of $225 \mathrm{Wm}^{-3}$, although on a small scale $(25 \mathrm{ml})$ and in a wellcontrolled lab environment [3].

Similarly to MFCs, state-of-the-art MECs suffer from low rates of production to volume ratio which has motivated the community to research improved reactor designs. [4].

Compared to the lab environment, not all operating conditions are observable or controllable in an industrial context. The wastewater composition typically varies uncontrollably while it has a major influence on the power generated (MFC) or the organics produced (MEC). Therefore, both require a run-time control scheme to automatically and dynamically determine the electrical settings for maximum power production or optimum organic production [5].

For MFCs, various maximum power point tracking algorithms have been developed [5]. The classic extremum seeking method uses a perturb \& observe scheme to derive the electrical load for which power is maximized [6]. Although this method is straightforward to implement, convergence speed is inverse proportional to accuracy and convergence takes very long in practical settings. More advanced methods [7]-[9] adapt the integrator gain based on a priori available calibration knowledge between the impact of the environment conditions and the maximum producible power. In [7], a second order relation is extracted between the inlet substrate concentration and the maximum power while [8] and [9] use a neural network (NN). Convergence is remarkably faster using this calibration knowledge. In practice however, the relation between the environment conditions and the maximum producible power is unknown and variable, thus requiring repetitive time-consuming on-line training.

A totally different method is the multi-unit optimization [10]. This method requires two or more identical MFCs, who operate with a minor offset on their operating settings between each other. From this offset, the gradient of the produced power is estimated using a finite difference approximation. Fast convergence occurs because the transients of the systems are compensated due to the multi-unit setup [11]. In practice it is however near impossible to have two identical setups. This mismatch is resolved with a time-consuming calibration method that characterizes the intrinsic MFC differences [12]. The repetitive time-consuming calibration due to the inherently slow, nonlinear, time-varying dynamics of MFCs [13] limits the practical adaptation of these controllers.

There are only a few control methodologies proposed for MECs [5]. In [14], the classical perturb \& observe scheme is used with the same drawback, i.e. very slow convergence, as with MFCs. In [15], a proportional integral derivative (PID) controller is used with adaptive gain to set the electrical settings. These optimal electrical settings are however derived from the optimization of a MEC model and thus only valid in a static environment.

This work presents a new discrete-based extremum seek- 


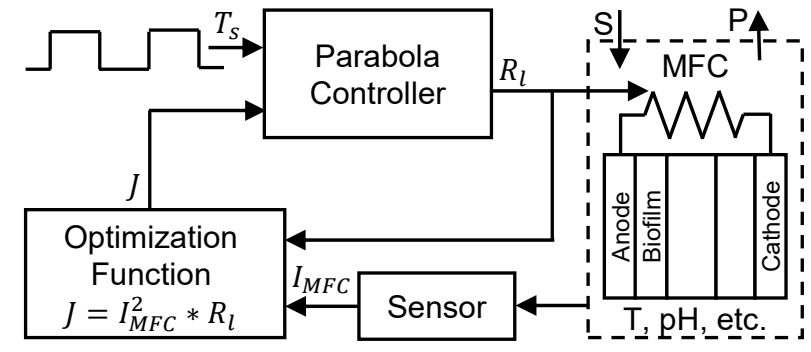

(a)

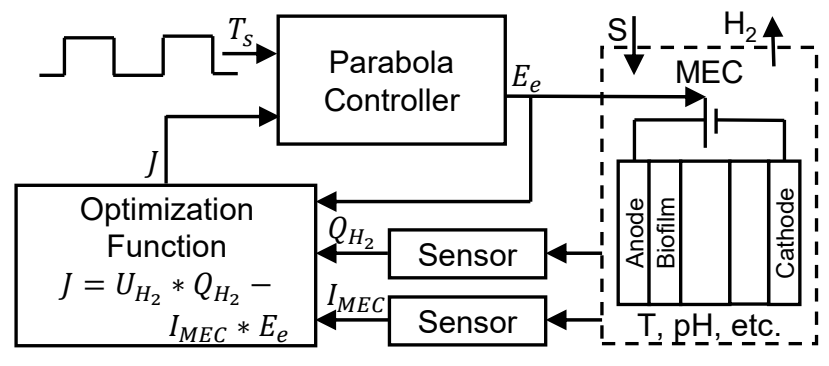

(b)

Fig. 1. Discrete extremum seeking control topology with sampling time $\left(T_{s}\right)$ for (a) a MFC, recovering electrical power (P) out of the inlet substrate concentration (S) and (b) a MEC, producing hydrogen gas $\left(\mathrm{H}_{2}\right)$ out of $\mathrm{S}$ at a certain temperature $(\mathrm{T}), \mathrm{pH}$, etc.

ing controller based on the numerical method of successive parabolic interpolation to automatically derive the optimal electrical settings for a flexible unimodal optimization function (Fig. 1). The control scheme treats the system as a black box, thus not requiring any calibration knowledge, and iteratively approximates the optimization function with a parabola. With minimal sampling points, and hence minimal convergence time, the algorithm converges to the optimum, both for a MFC or a MEC. Simulation studies show that convergence occurs $3 \mathrm{x}$ faster than state-of-the-art extremum seeking controllers without loss of precision of the optimal settings.

This paper is organized as follows. Section II describes the system, the discrete controller architecture and the optimization problem. Section III discusses in detail the operation of the discrete parabola controller. Section IV shows the results of the simulation study. Finally, section V compares the proposed technique with the current state-of-the-art.

\section{MAXIMIZING MFC POWER AND OPTIMIZING MEC ORGANIC PRODUCTION}

A redox reaction, whereby electrons are transported, occurs within both a MFC and a MEC. At the biotic anode, the catalytic activity of micro-organisms oxidizes the carbon of the organics to $\mathrm{CO}_{2}$. At the cathode of a $\mathrm{MFC}$, oxygen is reduced hereby releasing electrical power to the surrounding. At the cathode of a MEC however, no oxygen is present and, with the aid of a small amount of external power and possibly micro-organisms, high-value organics are formed. The most basic setup uses an abiotic cathode to produce hydrogen gas. An equivalent ion current flows through the liquid, separated by a membrane, to close the electrical loop. The generated power (MFC) or chemical production rate (MEC) strongly depends on the (assumed) controllable electrical settings and (assumed) uncontrollable operating conditions like inlet substrate concentration, temperature $(\mathrm{T}), \mathrm{pH}$, etc. For each of these conditions, our proposed discrete parabola controller determines the electrical settings (the load resistance $R_{l}$ ) for maximum power generation or (the applied external voltage $E_{e}$ ) for optimal chemical production rate (Fig. 1). The controller architecture is similar for a MFC and a MEC, although the optimization function is different.

This optimization problem is further specified based on the MFC model of [16], used for simulation and comparison. For a given MFC state $x \in \mathbb{R}^{4}$ (substrate, anodophilic microorganisms, methanogenic microorganisms and oxidized mediator fraction) and input $u \in \mathbb{R}^{2}$ (inlet substrate concentration $(S)$ and load resistance $\left(R_{l}\right)$ ), the constrained optimization problem becomes:

$$
\begin{array}{ll} 
& \max _{u} J_{M F C}(x, u) \\
\text { s.t. } & F_{M F C}(x, u)=0,
\end{array}
$$

where $J_{M F C}$ is the optimization function equaling the produced power (with maximum equaling $J_{M F C}^{*}$ ) and $F_{M F C}$ is the system dynamics. This constrained optimization problem is converted to an unconstrained one with minimal additional assumptions to guarantee convergence for the proposed iteration scheme. For each practical input $u$, we assume the system evolves to an asymptotic stable equilibrium point $x_{e q}(u)$ resulting in $F_{M F C}\left(x_{e q}(u), u\right)=0$. Because typically only $R_{l}$ is controllable, and $S$ is assumed constant during the extremum seeking, $J_{M F C}$ simplifies to 1-dimensional and unconstrained with an (unknown) parameter $S$ :

$$
J_{M F C}\left(x_{e q}(u), u\right)=I_{M F C}\left(x_{e q}\left(R_{l}, S\right), R_{l}, S\right)^{2} * R_{l},
$$

where $I_{M F C}$ is the produced current of the MFC. The discrete parabola controller uses this format of $J_{M F C}$ (Fig. 1a). Although $J_{M F C}$ could have any shape, we further limit $R_{l}$ to the interval for which $J_{M F C}$ is unimodal, defined according to [17] which corresponds with its practical domain for various $S$ (Fig. 2a). As has shown before in [7]-[9], $R_{l}$ that maximizes (2) depends on $S$.

An analogous derivation results in a similar optimization problem for a MEC, specified using a model from [18]. For state $x \in \mathbb{R}^{4}$ (substrate, anodophilic microorganisms, hydrogenotrophic microorganisms and mediator oxidized fraction) and input $u \in \mathbb{R}^{2}$ (inlet substrate concentration $(S$ ) and applied external voltage $\left.\left(E_{e}\right)\right)$, the optimization problem becomes:

$$
\begin{array}{ll} 
& \max _{u} J_{M E C}(x, u) \\
\text { s.t. } & F_{M E C}(x, u)=0,
\end{array}
$$

where $J_{M E C}$ is the net power balance (difference between the produced hydrogen enthalpy and invested input power [19]) (with maximum equaling $J_{M E C}^{*}$ ) and $F_{M E C}$ is the system 


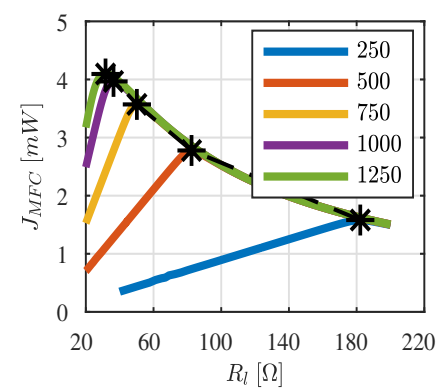

(a)

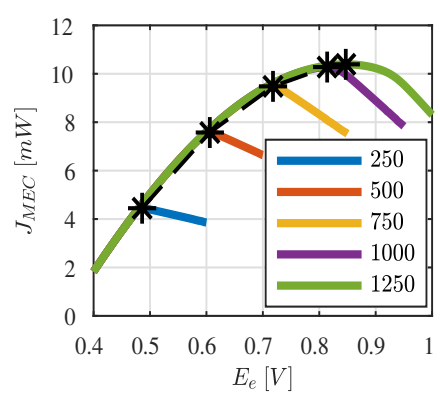

(b)
Fig. 2. (a) Generated output power of a MFC in function of $R_{l}$ and (b) net power balance of a MEC in function of $E_{e}$ for different $S\left[m g L^{-1}\right]$. The stars indicate the optimum.

dynamics. Making the same assumptions, $J_{M E C}$ simplifies to:

$$
\begin{aligned}
J_{M E C}\left(x_{e q}(u), u\right)= & U_{H_{2}} * Q_{H_{2}}\left(x_{e q}\left(E_{e}, S\right), E_{e}, S\right)- \\
& I_{M E C}\left(x_{e q}\left(E_{e}, S\right), E_{e}, S\right) * E_{e},
\end{aligned}
$$

where $U_{H_{2}}$ is the volumetric energy density of $H_{2}, Q_{H_{2}}$ is the produced volume $H_{2}$ per time unit and $I_{M E C}$ is the MEC current. The discrete parabola controller uses this format of $J_{M E C}$ (Fig. 1b). The applied external power maximizing (4) depends likewise on $S$ (Fig. 2b) and increases with a higher input substrate load.

\section{Successive Parabolic Interpolation as an Extremum Seeking Control Algorithm}

Extremum seeking control solves (1) and (3) assuming that the system transients disappear after a finite time. The classical approach applies a low-amplitude sinusoidal perturbation signal to the system. The gradient of the optimization function $J$ is then calculated from the high frequency content of the system response. This gradient is forced to zero by the integral feedback controller to maximize (1a) or (3a). The frequency of the perturbing signal is generally an order of magnitude lower than the dominant transient system response, which would otherwise affect this gradient. This is the cause of the slow convergence of this method [19].

To improve the convergence speed, this work proposes the successive parabolic interpolation algorithm from numerical mathematics as a discrete alternative to search $u^{*}$. This approach iteratively improves its estimation of the input to find the value of $R_{l}$ maximizing (2), respectively to find the value of $E_{e}$ maximizing (4). In every iteration step, the algorithm calculates a new estimate for which a fitted parabola through the previous 3 estimates reaches a maximum [17]. The iteration step ends by evaluating $J$ in this newly obtained input setting.

Evaluating $J$ is time-consuming because it involves a measurement on the actual MFC or MEC, for which (1b) respective (3b) must be fulfilled such that the system is in its steady state. The required sample time $T_{s}$ for each measurement is an upper bound for which $\left|x(t, u)-x_{e q}(u)\right|$

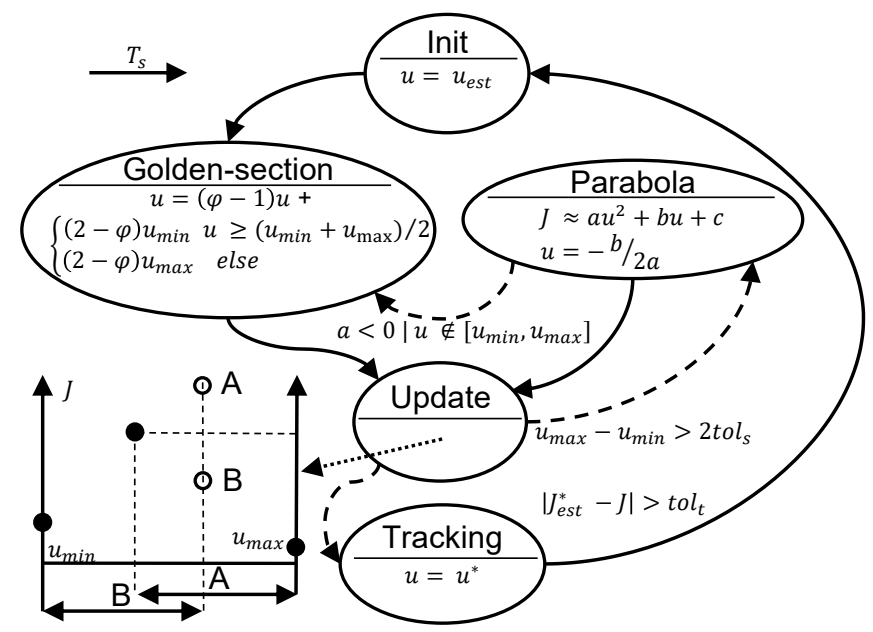

Fig. 3. Finite state machine of the discrete parabola controller. Solid arrows indicate a time-consuming transition of $T_{s}$. The golden ratio equals $\phi$. During the Update state, either part A or part B is kept of the search interval.

becomes sufficiently small for each potential $u$ of the search interval. For linear first order dynamics, $T_{s}=3 . .5 \tau$ with $\tau$ the dominant system time constant. The major benefit of this iteration scheme is guaranteed convergence, up to superlinear as $J$ is sufficiently smooth $\left(J \in C^{2}\right)$ (Fig. 2). This iteration scheme thus reduces the required amount of evaluations of $J$, which forms the dominant bottleneck for fast convergence.

The discrete parabola controller is implemented as a finite state machine (Fig. 3). During the initialization state (state Init in Fig. 3), the controller requires a bounded search interval $u_{\min }$ and $u_{\max }$ with $u_{\min }<u_{\max }$ such that $u_{\text {opt }}(S) \in$ $\left[u_{\min }, u_{\max }\right]$ for expected variations of $S$ and an initial estimate $u_{e s t} \in\left[u_{\min }, u_{\max }\right]$, the first measurement sample. Each successive $u$ is applied for a duration of $T_{s}$ to fulfill (1b) or (3b) and thus approximate $J$ using (2) or (4).

Golden-section search determines the next two samples because fitting a parabola requires at least 3 samples. The controller then goes to the Parabola state and approximates $J$ with a parabola. If no valid parabola is fitted, a save Goldensection step is performed. After sampling this new input, the controller reduces the search interval with the knowledge of the new sample during the Update state.

The controller moves to the Tracking state if the convergence criterion is met and records the discovered $J_{\text {est }}^{*}$. During this state, the controller keeps the input at $u^{*}$ and continuously re-samples $J$. If $\Delta J<t o l_{t}$, due to an external disturbance, the controller re-initializes the successive parabolic interpolation iteration scheme with the same initial search interval but using $u^{*}$ as initial estimator.

\section{Simulation Study}

The first set of simulations studies the iteration scheme of the discrete controller for a MFC model [16] and a MEC model [18]. Both models receive an inlet substrate concentration of $S=750 \mathrm{mgL}^{-1}$ and contain a full-grown biofilm. The configurable parameters of the controller with their value are 
TABLE I

Discrete Parabola Controller Parameters

\begin{tabular}{l|cc|cc|cc}
\hline & \multicolumn{2}{|c|}{ Iteration } & \multicolumn{2}{c|}{ Precise Tracking } & \multicolumn{2}{c}{ Fast Tracking } \\
\hline System & MFC & MEC & MFC & MEC & MFC & MEC \\
\hline$u_{\min }$ & $20 \Omega$ & $0.40 V$ & $20 \Omega$ & $0.40 V$ & $20 \Omega$ & $0.40 \mathrm{~V}$ \\
$u_{\text {est }}$ & $60 \Omega$ & $0.70 V$ & $60 \Omega$ & $0.70 \mathrm{~V}$ & $85 \Omega$ & $0.70 \mathrm{~V}$ \\
$u_{\max }$ & $100 \Omega$ & $1 \mathrm{~V}$ & $100 \Omega$ & $1 \mathrm{~V}$ & $100 \Omega$ & $1 \mathrm{~V}$ \\
$T_{s}$ & $1 \mathrm{day}$ & 1 day & 1 day & 1 day & 0.6 days & 0.5 days \\
tol $_{s}$ & $0.5 \%$ & $0.5 \%$ & $3.3 \%$ & $1.3 \%$ & $8.4 \%$ & $3.8 \%$ \\
tol $_{t}$ & - & - & $1 \%$ & $1 \%$ & $5 \%$ & $5 \%$ \\
\hline
\end{tabular}

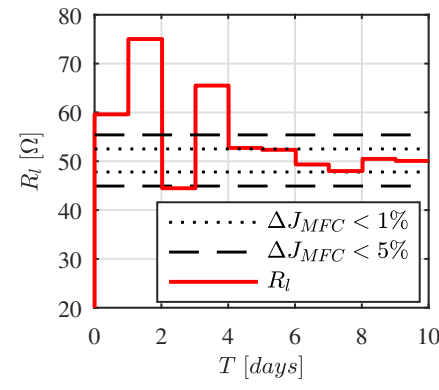

(a)

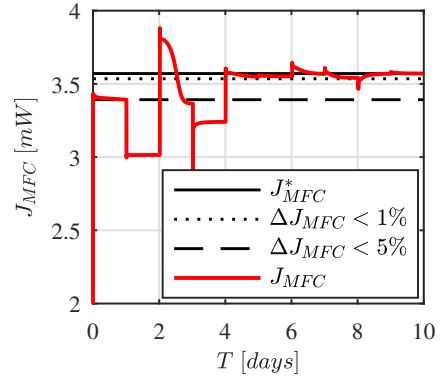

(b)
Fig. 4. (a) Applied $R_{l}$ and (b) corresponding $J_{M F C}$ for a possible iteration scheme of a MFC.

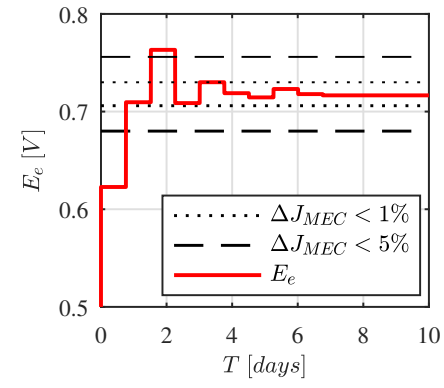

(a)

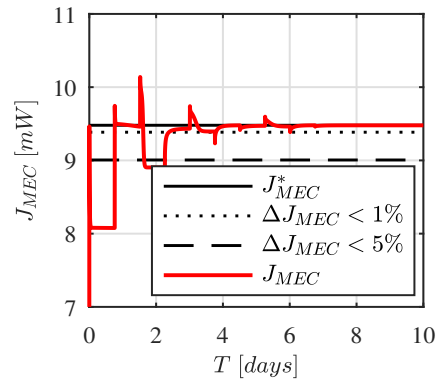

(b)
Fig. 5. (a) Applied $E_{e}$ and (b) corresponding $J_{M E C}$ for a possible iteration scheme of a MEC.

given in Table I, under column Iteration. The initial search interval follows from Fig. $2 \mathrm{a}$ and Fig. $2 \mathrm{~b}$, the initial estimate $u_{\text {est }}$ is chosen near the middle and the sample time $T_{s}$ and convergence tolerance $t_{o l}$ settings follow from the discussion of the next paragraph. A typical iteration scheme is shown in Fig. 4 for a MFC and in Fig. 5 for a MEC. After the controller has applied a new sampling input, the optimization function sometimes surpasses the true maximum at the beginning of the sample interval due to the system transients.

The next set of simulations studies the convergence speed and resulting precision of the discrete controller. Both are dependent on the initial search interval $\left[u_{\min }, u_{\max }\right], u_{e s t}$ and $T_{s}$. The search interval and the estimate encapsulate the a priori knowledge about the system and should be taken as precise as possible. The sample time however influences the impact of the dynamics of the system on $J$. Convergence is guaranteed theoretically, only if $T_{s} \rightarrow \infty$ such that (2)

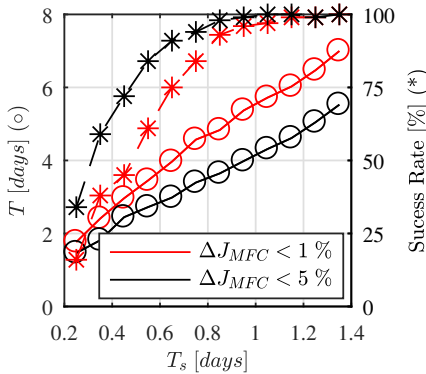

(a)

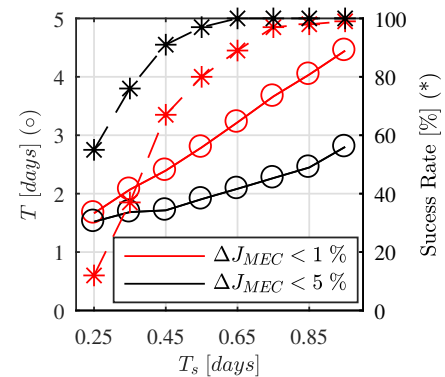

(b)
Fig. 6. Convergence time (o, left) and success rate (*, right) of the iteration scheme for (a) a MFC and (b) a MEC for a linear sweep of initial estimates in function of $T_{s}$.

approximates to (1) and (4) to (3), respectively. In practice, $T_{s}$ can be chosen to be smaller at the expense of a loss in accuracy, which results in a trade-off between speed and precision. Fig. 6a demonstrates the resulting trade-off for a MFC and Fig. 6b for a MEC. Every dot on this graph is obtained by, for a particular $T_{s}$, running the parabola controller for 100 different, linearly varied, initial estimates. All other controller configuration settings are taken from the parameters from Table I. The parameter $t_{\text {ol }}$ is set to stabilize within $1 \%$ or $5 \%$ of $J^{*}$. Depending on the length of $T_{s}$ and $u_{e s t}$, convergence up to the target precision is now no longer guaranteed. This can be observed from the success rate curves in Fig. 6a and Fig. 6b. Very fast convergence, $<3$ days for a MFC and $<2$ days for a MEC, is possible, however, at a cost of a lower likelihood $(90 \%)$ for the initial estimate to achieve a precision of $\Delta J<5 \%$. The appropriate sample time should therefore be configured depending on the frequency of the expected variation of the inlet substrate concentration and the desired tolerance on the optimization function.

The last set of simulations studies the tracking capabilities of the discrete parabola controller. The inlet substrate concentration is first varied every 10 days with the following pattern: $S\left[\mathrm{mgL}^{-1}\right]=500 \rightarrow 1000 \rightarrow 500 \rightarrow 750 \rightarrow$ $1250 \rightarrow 1000 \rightarrow 500$. The tolerance parameters of the discrete parabola controller are configured to achieve an equivalent $\Delta J$ precision of $<1 \%$ (Table I, under column Precise Tracking). Fig. 7 for a MFC and Fig. 8 for a MEC show the obtained input determined by the controller focusing on precise tracking of the inlet substrate variation. After convergence in around 6 days, the controller continuously re-samples $J$. If $J$ varies with more than $t_{0} l_{t}$, the iteration scheme restarts with the previous optimum as initial estimate. Because the controller has no knowledge of the substrate concentration, it does not know if the input should rather increase or decrease. Therefore, Golden-section search determines the first two observed inputs which could lie in the opposite direction.

In the last simulation, Fig. 9 and Fig. 10, the substrate variation pattern of the previous simulation is applied every 4 days. The controller parameters are modified to focus on fast convergence rather than high precision (Table I, under column 


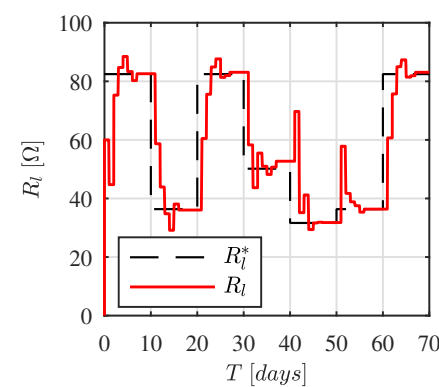

(a)

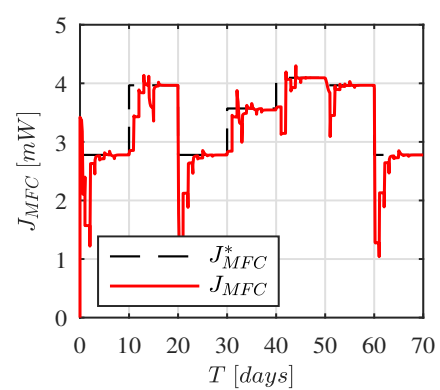

(b)
Fig. 7. (a) Applied $R_{l}$ and (b) corresponding $J_{M F C}$ of a MFC for a $S$ variation every 10 days with the parabola controller focusing on precision.

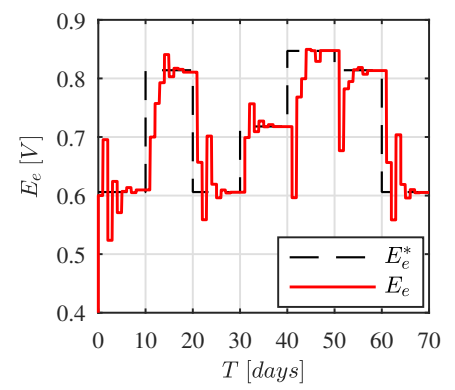

(a)

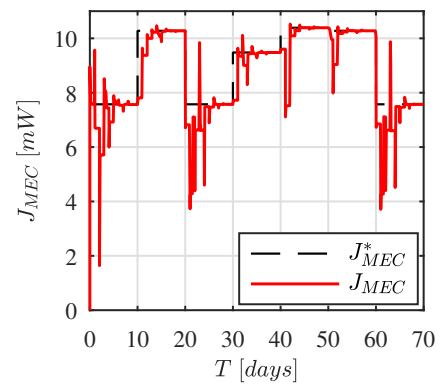

(b)
Fig. 8. (a) Applied $E_{e}$ and (b) corresponding $J_{M E C}$ of a MEC for a $S$ variation every 10 days with the parabola controller focusing on precision.

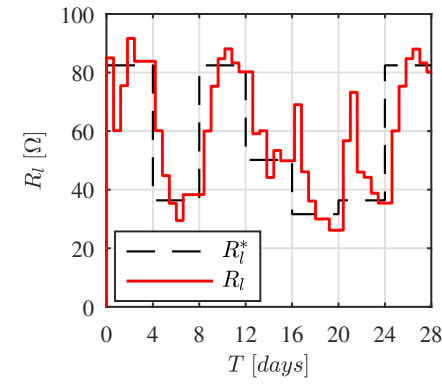

(a)

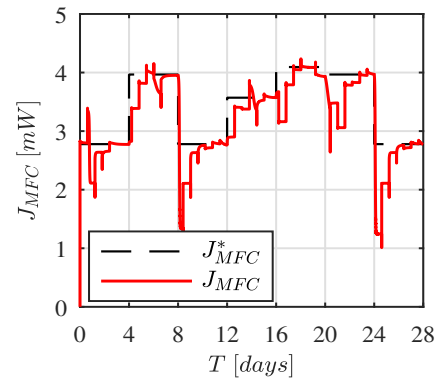

(b)
Fig. 9. (a) Applied $R_{l}$ and (b) corresponding $J_{M F C}$ of a MFC for a $S$ variation every 4 days with the parabola controller focusing on fast convergence.

Fast Tracking). The controller now converges in about 3 days to the optimum, however at the cost of a reduced precision, $\Delta J<5 \%$. This convergence speedup (and corresponding loss of precision) is due to a lower $T_{s}$ and thus a worse satisfaction of the constraints (1b) and (3b).

\section{DISCUSSION}

The benefits of tracking the optimum input compared to merely applying a static input for dynamic environment conditions is illustrated with the inlet substrate variation example of the previous section (Table II). The discrete parabola controller achieves $4 \%$ to $11 \%$ more performance of the maximum achievable performance compared to applying the average of

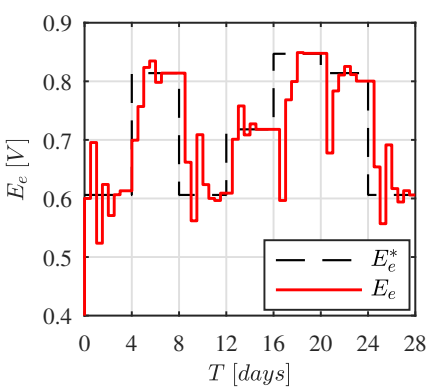

(a)

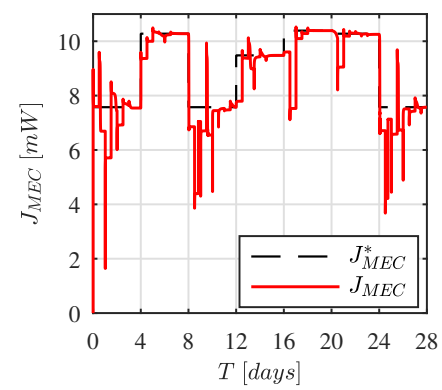

(b)
Fig. 10. (a) Applied $E_{e}$ and (b) corresponding $J_{M E C}$ of a MEC for a $S$ variation every 4 days with the parabola controller focusing on fast convergence.

TABLE II

RELATIVE PERFORMANCE FOR VARIOUS STATIC INPUTS AND THE TRACKING INPUT OF THE DISCRETE PARABOLA CONTROLLER

\begin{tabular}{l|c|c|c|c}
\hline Experiment & $u_{\min }$ & $u_{\max }$ & $u_{\text {avg }}$ & Tracking \\
\hline MFC, 10 days $\Delta S$ & $56 \%$ & $74 \%$ & $84 \%$ & $\mathbf{9 5} \%$ \\
MEC, 10 days $\Delta S$ & $20 \%$ & $81 \%$ & $91 \%$ & $\mathbf{9 7} \%$ \\
MFC, 4 days $\Delta S$ & $56 \%$ & $74 \%$ & $85 \%$ & $\mathbf{9 4} \%$ \\
MEC, 4 days $\Delta S$ & $20 \%$ & $81 \%$ & $92 \%$ & $\mathbf{9 6} \%$ \\
\hline
\end{tabular}

the optimal inputs. The rather small difference between the precise configuration and the fast configuration is because the temporal suboptimal inputs during convergence towards the new optimum input have a larger impact on the configuration for precise operation than for fast operation.

Table III compares this work with the state-of-the-art on extremum seeking control for MFCs. It is important to note that all reported convergence times in the table are assessed on the same MFC model, given by [16]. The classical method of perturb \& observe converges extremely slow ( \pm 100 days) due to the inherent slow dynamics of a MFC [9]. This method however achieves a good precision $(<1 \%)$ and does not require any a priori calibration nor extra sensors besides the ones for calculating the optimization function $J$. The stability condition further limits the integral feedback gain and thus fast convergence.

To resolve this slow convergence, the state-of-the-art adds an extra feedback path to increase the integral gain based on an a priori calibrated relation between the inlet substrate $(S)$ variation and the optimum input. In [7], this relation between $R_{l}$ and $S$ is fitted using a second order polynomial (Pol). Adding this knowledge to the feedback path results in a $10 \mathrm{x}$ convergence speedup, however at the cost of a $5 \mathrm{x}$ loss of precision. Approximating this relation with a NN results in $5 \mathrm{x}$ better precision, with a minor impact on convergence speed [8], [9]. The major drawback of these controllers is the requirement of calibrated data of the MFC for the optimal input for a given set of $S$ or, more general, for every, expected environment condition that influences $J$, an unrealistic assumption for a practical setup. Obtaining this calibration data in real-time is not specified but nevertheless requires a time-consuming procedure due to the steady-state requirement for the numerous 
TABLE III

COMPARISON OF THIS WORK WITH PREVIOUS PUBLISHED EXTREMUM SEEKING CONTROLLERS

\begin{tabular}{|c|c|c|c|c|c|}
\hline Reference & Perturb \& Observe [9] & IECON'12 [7] & MED'15 [8] & IECON'16 [9] & $\begin{array}{c}\text { This Work } \\
\end{array}$ \\
\hline Convergence for [16] & \pm 100 days & \pm 10 days & \pm 60 days & \pm 15 days & 3.5 days $\pm 0.8 \sigma \quad 5.4$ days $\pm 1.4 \sigma$ \\
\hline Prec & $<1 \%$ & $<5 \%$ & $<1 \%$ & $<1 \%$ & $<\mathbf{5} \%$ \\
\hline Calib & - & $R_{l}=\operatorname{Pol}(S)$ & $R_{l}=N N(S)$ & $R_{l}=N N(S)$ & - \\
\hline Requir & $J$ & $J, S$ & $J, S$ & $J, S$ & $\boldsymbol{J}$ \\
\hline $\begin{array}{l}\text { Environment Variation } \\
\text { Stability }\end{array}$ & $\begin{array}{c}S, T, p H, \text { etc... } \\
\text { not guaranteed }\end{array}$ & $\begin{array}{c}S \\
\text { not guaranteed }\end{array}$ & $\begin{array}{c}S \\
\text { not guaranteed }\end{array}$ & $\begin{array}{c}S \\
\text { not guaranteed }\end{array}$ & $\begin{array}{c}S, T, p H \text {, etc... } \\
\text { inherently stable }\end{array}$ \\
\hline
\end{tabular}

measurement samples.

The proposed discrete parabola controller converges on average in 3.5 days $\pm 0.8 \sigma$ for a likelihood of $95 \%$ that the initial estimate results in a precision of $\Delta J<5 \%$ and on average in 5.4 days $\pm 1.4 \sigma$ for a likelihood of $95 \%$ that the initial estimate results in a precision of $\Delta J<1 \%$, a 3x improvement in convergence speed compared to the state-of-the-art for an equal precision on the model of [16]. Furthermore, no calibration is required besides a rough estimate of the search interval. The controller is inherently stable with guaranteed convergence if $T_{s}$ is chosen such that (1b) or (3b) are met. Finally, the controller is not limited to variations in the inlet substrate concentration nor to a MFC.

\section{CONCLUSION}

A discrete extremum seeking controller is designed to automatically and dynamically determine the electrical settings for optimal operation of a MFC and a MEC irrespective of the environment conditions. This discrete controller uses the successive parabolic interpolation iteration scheme to converge quickly to the optimum input with a minimum of sampling points. The parameters of the controller are configurable to either focus on fast convergence or on high precision.

For an established MFC model, convergence occurs in about 3.5 days to stabilize within $5 \%$ of the cell's maximum performance and in about 5.4 days to stabilize within $1 \%$ of the cell's maximum performance. Equal convergence speeds are achieved for a MEC model. Convergence is guaranteed for an appropriate sample time with respect to the system time constant and an initial search interval containing the optimum.

The dominant time required for convergence of this discrete parabola controller is the static sample time. Therefore, further research should explore the benefits of a dynamic sample time.

\section{ACKNOWLEDGMENT}

This work was supported by a Doctoral Fellowship of the Research Foundation Flanders (FWO). We would like to thank Athanasios Sarafianos, Bert Moons and Sander Smets for review and discussion.

\section{REFERENCES}

[1] B. E. Logan and K. Rabaey, "Conversion of wastes into bioelectricity and chemicals by using microbial electrochemical technologies," Science, vol. 337, no. 6095, pp. 686-690, 2012.

[2] A. D. Tharali, N. Sain, and W. J. Osborne, "Microbial fuel cells in bioelectricity production," Frontiers in Life Sci., vol. 9, no. 4, pp. 252266,2016
[3] Y. Yang, T. Liu, X. Zhu, F. Zhang, D. Ye, Q. Liao, and Y. Li, "Boosting power density of microbial fuel cells with 3D nitrogen-doped graphene aerogel electrode," Advanced Sci., vol. 3, no. 8, 2016.

[4] A. Kadier, Y. Simayi, P. Abdeshahian, N. F. Azman, K. Chandrasekhar, and M. S. Kalil, "A comprehensive review of microbial electrolysis cells (MEC) reactor designs and configurations for sustainable hydrogen gas production," Alexandria Eng. J., vol. 55, no. 1, pp. 427 - 443, 2016.

[5] D. Recio-Garrido, M. Perrier, and B. Tartakovsky, "Modeling, optimization and control of bioelectrochemical systems," Chemical Eng. J., vol. 289, pp. $180-190,2016$.

[6] L. Woodward, M. Perrier, B. Srinivasan, R. P. Pinto, and B. Tartakovsky, "Comparison of real-time methods for maximizing power output in microbial fuel cells," AIChE J., vol. 56, no. 10, pp. 2742-2750, 2010.

[7] S. Attarsharghi, L. Woodward, and O. Akhrif, "An improved maximum power extraction scheme for microbial fuel cells," in IECON 2012 - 38th Annu. Conference on IEEE Ind. Electron. Soc., 2012, pp. 910-915.

[8] A. Kebir, L. Woodward, and O. Akhrif, "Extremum-seeking control with anticipative action of microbial fuel cell's power," in 2015 23rd Mediterranean Conference on Control and Automation (MED), 2015, pp. 933-939.

[9] A. Kebir, O. Akhrif, and L. Woodward, "Extremum-seeking control of a microbial fuel cell power using adaptive excitation," in IECON 2016 - 42nd Annu. Conference of the IEEE Ind. Electron. Soc., 2016, pp. 4127-4132.

[10] B. Srinivasan, "Real-time optimization of dynamic systems using multiple units," Int. J. of Robust and Nonlinear Control, vol. 17, no. 13, pp. 1183-1193, 2007.

[11] L. Woodward, B. Tartakovsky, M. Perrier, and B. Srinivasan, "Maximizing power production in a stack of microbial fuel cells using multiunit optimization method," Biotechnology Progress, vol. 25, no. 3, pp. 676682, 2009.

[12] L. Woodward, M. Perrier, and B. Srinivasan, "Improved performance in the multi-unit optimization method with non-identical units," $J$. of Process Control, vol. 19, no. 2, pp. 205 - 215, 2009.

[13] F. Zhao, R. C. T. Slade, and J. R. Varcoe, "Techniques for the study and development of microbial fuel cells: an electrochemical perspective," Chem. Soc. Rev., vol. 38, pp. 1926-1939, 2009.

[14] B. Tartakovsky, P. Mehta, G. Santoyo, and S. Guiot, "Maximizing hydrogen production in a microbial electrolysis cell by real-time optimization of applied voltage," Int. J. of Hydrogen Energy, vol. 36, no. 17, pp. 10557 - 10564, 2011.

[15] A. M. Yahya, M. A. Hussain, and A. K. Abdul Wahab, "Modeling, optimization, and control of microbial electrolysis cells in a fed-batch reactor for production of renewable biohydrogen gas," Int. J. of Energy Research, vol. 39, no. 4, pp. 557-572, 2015.

[16] R. Pinto, B. Srinivasan, M.-F. Manuel, and B. Tartakovsky, "A twopopulation bio-electrochemical model of a microbial fuel cell," Bioresource Technology, vol. 101, no. 14, pp. 5256 - 5265, 2010.

[17] R. P. Brent, Algorithms for Minimization without Derivatives. Englewood Cliffs, New Jersey: Prentice-Hall, Inc., 1973.

[18] R. Pinto, B. Srinivasan, and B. Tartakovsky, "A unified model for electricity and hydrogen production in microbial electrochemical cells," IFAC Proc. Volumes, vol. 44, no. 1, pp. 5046 - 5051, 2011.

[19] R. Pinto, B. Tartakovsky, and B. Srinivasan, "Optimizing energy productivity of microbial electrochemical cells," J. of Process Control, vol. 22, no. 6, pp. 1079 - 1086, 2012. 